\title{
Effects of Extracorporeal Membrane Oxygenation Initiation on Oxygenation and Pulmonary Opacities
}

\author{
Kiran Batra, Manish Mohanka, Srinivas Bollineni, Vaidehi Kaza, Prabhakar Rajiah, Yin Xi, \\ Amy Hackmann, Michael Wait, Fernando Torres, Amit Banga* \\ University of Texas Southwestern Medical Center at Dallas, TX, USA
}

\begin{abstract}
Introduction: There is limited data on the impact of extracorporeal membrane oxygenation (ECMO) on pulmonary physiology and imaging in adult patients. The current study sought to evaluate the serial changes in oxygenation and pulmonary opacities after ECMO initiation. Methods: Records of patients started on veno-venous, or veno-arterial ECMO were reviewed ( $n=33$; mean (SD): age 50(16) years; Male: Female 20:13). Clinical and laboratory variables before and after ECMO, including daily $\mathrm{PaO}_{2}$ to $\mathrm{FiO}_{2}$ ratio (PFR), were recorded. Daily chest radiographs (CXR) were prospectively appraised in a blinded fashion and scored for the extent and severity of opacities using an objective scoring system. Results: ECMO was associated with impaired oxygenation as reflected by the drop in median PFR from 101 (interquartile range, IQR: 63-151) at the initiation of ECMO to a post-ECMO trough of 74 (IQR: 56-98) on post-ECMO day 5 . However, the difference was not statistically significant. The appraisal of daily CXR revealed progressively worsening opacities, as reflected by a significant increase in the opacity score (Wilk's Lambda statistic 7.59, $p=0.001$ ). During the post-ECMO period, a $>10 \%$ increase in the opacity score was recorded in $93.9 \%$ of patients. There was a negative association between PFR and opacity scores, with an average one-unit decrease in the PFR corresponding to a +0.010 increase in the opacity score (95\% confidence interval: 0.002 to $0.019, p$-value $=0.0162$ ). The median opacity score on each day after ECMO initiation remained significantly higher than the pre-ECMO score. The most significant increase in the opacity score (9, IQR: -8 to 16$)$ was noted on radiographs between pre-ECMO and forty-eight hours post-ECMO. The severity of deteriorating oxygenation or pulmonary opacities was not associated with hospital survival. Conclusions: The use of ECMO is associated with an increase in bilateral opacities and a deterioration in oxygenation that starts early and peaks around 48 hours after ECMO initiation.
\end{abstract}

Keywords: ECMO, acute lung injury, cytokine surge, refractory hypoxia, systemic inflammatory response

Received: 9 July 2020 / Accepted: 27 October 2020

\section{INTRODUCTION}

The use of extracorporeal membrane oxygenation (ECMO) as a strategy to support adult patients with refractory hypoxemia or hypercapnia as well as for hemodynamic support, continues to increase [1]. Given the temporary nature of the ECMO support for pulmonary indications, it is considered a 'bridge to destination' therapy. ECMO may aid in the decision-making process, though ultimately, patients were bridged to either recovery or lung transplantation (LT) surgery.

Despite significant advancements in the pump and circuitry equipment used for ECMO, which allowed patients to be supported for more extended periods, it is not a benign intervention, and the clinical course among patients on ECMO can be precarious with the potential for complications to follow [2]. Several research studies, mostly conducted in paediatric populations, have demonstrated that the initiation of ECMO, almost immediately activates an inflammatory cascade, similar to the systemic inflammatory response syndrome (SIRS) [3-10]. This cascade leads to the release of several pro-inflammatory cytokines that causes organ dysfunction.

Despite pre-clinical studies demonstrating the proinflammatory effects of ECMO initiation, there remains a lack of data regarding the frequency of these complications and their impact on outcomes among adult patients on ECMO. 
Specifically, data are lacking regarding the pulmonary effects of ECMO related SIRS, which can be hard to differentiate from the background lung disease necessitating ECMO support.

The current study aimed to evaluate the progressive changes in oxygenation and pulmonary opacities after the initiation of ECMO, correlate these changes with non-pulmonary organ functions and evaluate its association with hospitalisation durations.

\section{- METHODS}

The Institutional Review Board of the University of Texas Southwestern Medical Centre in Dallas, Texas, USA, provided approval for the study with the waiver that written patient consent be obtained before the commencement of the study (IRB\# STU 072015-001).

The institutional ECMO database of the centre was searched. Patients with advanced lung disease who needed VV or VA ECMO during three years between 1st January 2013 and 31st December 2015 were eligible for inclusion $(n=37)$. Among these, patients who did not survive at least 96 hours after ECMO initiation were excluded $(n=4)$.

\section{Extracorporeal life support}

All patients were on veno-venous (VV) or veno-arterial (VA) ECMO for respiratory failure $(n=33)$. The mean (SD) age was 50 (16) years (range 14-69 years; M: F 20:13).

The goals for the ECMO support for each patient in the study group were classified into "bridge to recovery "( $\mathrm{n}=9)$ and "bridge to transplantation" $(\mathrm{n}=24)$.

Details of patients' demographics, clinical and laboratory variables before and after initiation of ECMO, daily $\mathrm{PaO}_{2} / \mathrm{FiO}_{2}(\mathrm{P} / \mathrm{F})$ ratios, chest radiographs, and development of organ dysfunction such as renal, hepatic, cardiac/hemodynamic, neurological and hematologic during the ECMO support were obtained from the records. The diagnosis of organ dysfunction was based upon the standard guidelines and was made by analysing the clinical and laboratory findings noted in the patient records [11].

The records showed that all patients included in the study had the following:

They had undergone a baseline echocardiogram to assess cardiac function before ECMO initiation and subsequently as clinically indicated. Patients on VAECMO had an arterial line placed on the contralateral radial artery, from the side of the arterial limb of the
VA-ECMO cannula, for continuous measurement of blood pressure and blood gas analysis. They had been maintained on unfractionated heparin drip at rates titrated to activated clotting times (ACT) between 140180 seconds for VV-ECMO and 180-220 seconds for VA-ECMO. They had been intubated and supported on mechanical ventilation at the time of ECMO initiation and had been transitioned to lung-protective ventilator strategies after the initiation of ECMO. Lung protective strategy included using low tidal volumes (6-8 $\mathrm{ml} / \mathrm{Kg}$ IBW) while maintaining plateau pressures $<30$ $\mathrm{cm} \mathrm{H}_{2} \mathrm{O}$. The level of PEEP had been titrated to enable $\mathrm{FiO}_{2}$ wean while ensuring the plateau pressures remained below $30 \mathrm{~cm} \mathrm{H}_{2} \mathrm{O}$. The duration of ECMO support, along with hospital survival for all patients, was recorded from the patient records.

Scoring was done on chest radiographs (CXR) taken before the ECMO initiation and the daily morning CXR, taken during seven days when patients were on ECMO during the study period. The chest radiographs were appraised and scored objectively for the study. All $\mathrm{X}$-rays were evaluated and scored by two thoracic radiologists, each with more than five years of experience. Both radiologists were blinded to the clinical data.

The radiographs were scored for the extent and severity of opacities using an objective scoring system that was based on the quality of the radiographs as well as the location, severity, and characteristics of the opacities (Table 1).

The 'opacity score' scoring system ranged from 0-25 for each lung. The opacity characteristics were scored as an incremental change in comparison to the baseline, pre-ECMO radiographs, which was assigned a score of zero for the opacity characteristics given the variable extent of the interstitial and alveolar opacities due to the background lung disease.

For the serial assessment, the addition or subtraction to the earlier score was made based on the extent and characteristics of opacities on the subsequent CXR, as described in Table 1.

Each radiologist scored the images independently, and any differences were reconciled.

\section{Statistical Analysis}

Interclass correlation (ICC) was used to assess for agreement in the opacity score. A linear mixed model was used to test the association between the $\mathrm{PaO}_{2} / \mathrm{FiO}_{2}$ ratio and opacity scores while adjusting for patient and time random effects. The change in opacity score with 
Table 1. Scoring system for chest radiographs for the extent and severity of opacities

\begin{tabular}{|c|c|c|c|c|c|}
\hline & \multicolumn{5}{|l|}{ Assigned Score } \\
\hline & 0 & 1 & 2 & 3 & 4 \\
\hline Quality & Nondiagnostic & Suboptimal & Diagnostic & & \\
\hline $\begin{array}{l}\text { Localization (Each lung di- } \\
\text { vided into upper, middle and } \\
\text { lower zones) }\end{array}$ & None & One zone & Two zones & Three zones & \\
\hline $\begin{array}{l}\text { Severity (Each lung field } \\
\text { divided into four quadrants) }\end{array}$ & None & One quadrant & Two quadrants & Three quadrants & $\begin{array}{l}\text { Four } \\
\text { quadrants }\end{array}$ \\
\hline Opacity characteristics & $\begin{array}{l}\text { No change } \\
\text { from baseline }\end{array}$ & $\begin{array}{l}\text { Hazy homoge- } \\
\text { neous opacities } \\
\text { without obscu- } \\
\text { ration of lung } \\
\text { markings with } \\
\text { or without air } \\
\text { bronchograms }\end{array}$ & $\begin{array}{l}\text { Confluent alveolar } \\
\text { and/or interstitial } \\
\text { opacities with } \\
\text { obscuration of } \\
\text { lung markings } \\
\text { with or without air } \\
\text { bronchograms }\end{array}$ & $\begin{array}{l}\text { Increasing at- } \\
\text { tenuation with } \\
\text { obscuration of } \\
\text { the mediastinal, } \\
\text { hemidiaphragms, } \\
\text { costophrenic } \\
\text { sulcus margins }\end{array}$ & $\begin{array}{l}\text { Complete } \\
\text { Whiteout }\end{array}$ \\
\hline
\end{tabular}

a 1-unit change in $\mathrm{PF}$ ratio was also estimated, with a 95\% confidence interval.

Changes in the median opacity scores from the preECMO radiographs, to those up to seven days after ECMO, along with the concurrent PF ratio, were analysed to study the trends after ECMO initiation. Patients were divided into two groups based on a 'significant' increase in their peak opacity score after ECMO initiation. Group 1 was comprised of patients with an incremental change in the opacity score, from baseline, in the highest quartile or top 25 percentile; Group 2 was comprised of patients with the opacity score change in the lower three quartiles or $<75$ percentile. Pre and post-ECMO parameters and outcomes variables were compared among the two groups using chi-square analysis and Mann Whitney $\mathrm{U}$ test as appropriate.

The level of statistical significance was set at $\alpha=0.05$.

\section{RESULTS}

\section{Study Cohort}

The majority of the patients were on ECMO as a bridge to transplant surgery $(72.7 \%)$.

Interstitial lung disease was the most common underlying disease (20/33, 60.6\%), necessitating ECMO support.

The majority of patients were on VV-ECMO support $(\mathrm{n}=28,84.8 \%)$ via Avalon in the right internal jugular vein $(n=21)$.

Five patients were on VA-ECMO due to concomitant right heart failure. These patients had evidence of a severely dilated right ventricle with reduced function as evidenced by the visual estimate and reduction in tricuspid annular plane systolic excursion $<16 \mathrm{~mm}$. Femoral-femoral cannulation was the most frequent strategy for the institution of VA-ECMO $(n=3)$.

\section{Oxygenation and Opacity score}

ECMO was associated with worsened oxygenation as reflected by a drop in median PF ratio from 101 (interquartile range, IQR: 63-151) at the initiation of ECMO to a post-ECMO trough of 74 on day 5 (IQR: 56-98) although the difference was statistically not-significant.

The review of chest radiographs revealed progressively worsening opacities, as reflected by a statistically significant increase in the opacity score (Wilk's Lambda statistic 7.59, $\mathrm{p}=0.001)$.

Most patients (93.9\%) had at least a $10 \%$ increase in their opacity score at some point during their post-EC$\mathrm{MO}$ course. There was a negative association between the PF ratio and opacity score with a one-unit decrease in the $\mathrm{PF}$ ratio associated with a 0.010 increase in opacity score (95\% CI: 0.002-0.019; $\mathrm{p}=0.0162)$ after adjustment.

Serial measurements of daily PF ratio before and after ECMO initiation are presented in Figure 1, while Figure 2 shows a representative patient with findings from serial chest radiographs.

On post hoc analysis, the median opacity score on each day after ECMO initiation remained significantly higher than the pre-ECMO scores. The opacity score peaked around 48 hours after ECMO initiation, beyond which it appeared to plateau while remaining consistently higher than the pre-ECMO score. 
Table 2. Baseline characteristics and outcomes among patients with and without the significant worsening in opacity score

\begin{tabular}{|c|c|c|c|}
\hline \multirow{2}{*}{ Variable } & \multicolumn{2}{|c|}{ Significant worsening in opacity score } & \multirow{2}{*}{ P-valuet } \\
\hline & Yes $(n=9)$ & No $(n=24)$ & \\
\hline Age & $56(50-69)$ & $53.5(14-66)$ & 0.11 \\
\hline Male sex & $55.6 \%$ & $62.5 \%$ & 0.72 \\
\hline Caucasian & $44.4 \%$ & $62.5 \%$ & 0.26 \\
\hline \multicolumn{4}{|l|}{ Underlying diagnosis necessitating ECMO } \\
\hline Interstitial lung disease & $77.8 \%$ & $54.2 \%$ & \multirow{3}{*}{0.64} \\
\hline ARDS & $11.1 \%$ & $16.7 \%$ & \\
\hline Others & $11.1 \%$ & $29.1 \%$ & \\
\hline Pre-ECMO PF ratio (mm Hg) & $99(84-336)$ & $88(64-164)$ & 0.31 \\
\hline Co-morbid pulmonary hypertension & $33.3 \%$ & $16.7 \%$ & 0.36 \\
\hline Pre-ECMO transfusion & $11.1 \%$ & $25 \%$ & 0.35 \\
\hline Pre-ECMO pressors & $33.3 \%$ & $54.2 \%$ & 0.35 \\
\hline Pre-ECMO use of inhaled nitric oxide & $88.9 \%$ & $62.5 \%$ & 0.18 \\
\hline Pre-ECMO positive blood cultures & None & None & \\
\hline VV-ECMO & $88.9 \%$ & $83.3 \%$ & 0.69 \\
\hline Intended Bridge to LT & $77.8 \%$ & $70.8 \%$ & 0.69 \\
\hline Post-ECMO transfusion & $88.9 \%$ & $79.2 \%$ & 0.52 \\
\hline Post-ECMO pressors & $66.7 \%$ & $87.5 \%$ & 0.17 \\
\hline Post-ECMO use of inhaled nitric oxide & $66.7 \%$ & $54.2 \%$ & 0.6 \\
\hline Post-ECMO fluid balance day 1-3 (L) & $+1.0(-1.8$ to +8.1$)$ & $+1.4(-6.5$ to +8.6$)$ & 0.59 \\
\hline Able to get out of bed on ECMO & $22.2 \%$ & $37.5 \%$ & 0.28 \\
\hline Able to ambulate on ECMO & None & $20.8 \%$ & 0.1 \\
\hline Successful bridge to LT & $57.1 \%(n=7)$ & $58.8 \%(n=17)$ & 0.94 \\
\hline Successful bridge to recovery & None $(n=2)$ & $57.1 \%(n=7)$ & 0.4 \\
\hline Duration of ECMO & $5(3-17)$ & $10(4-47)$ & 0.047 \\
\hline Hospital Survival & $55.5 \%$ & $58.3 \%$ & 1.0 \\
\hline
\end{tabular}

Also, the change in the median opacity score was the highest among radiographs taken between pre-ECMO and 48 hours post-ECMO (9, IQR: -8 to 16 , Figure 1 ).
The characteristics and outcomes among patients with a significant change in the opacity score $(>16, n=9)$ and those without $(\leq 16, \mathrm{n}=24)$ are presented in Table 2 .

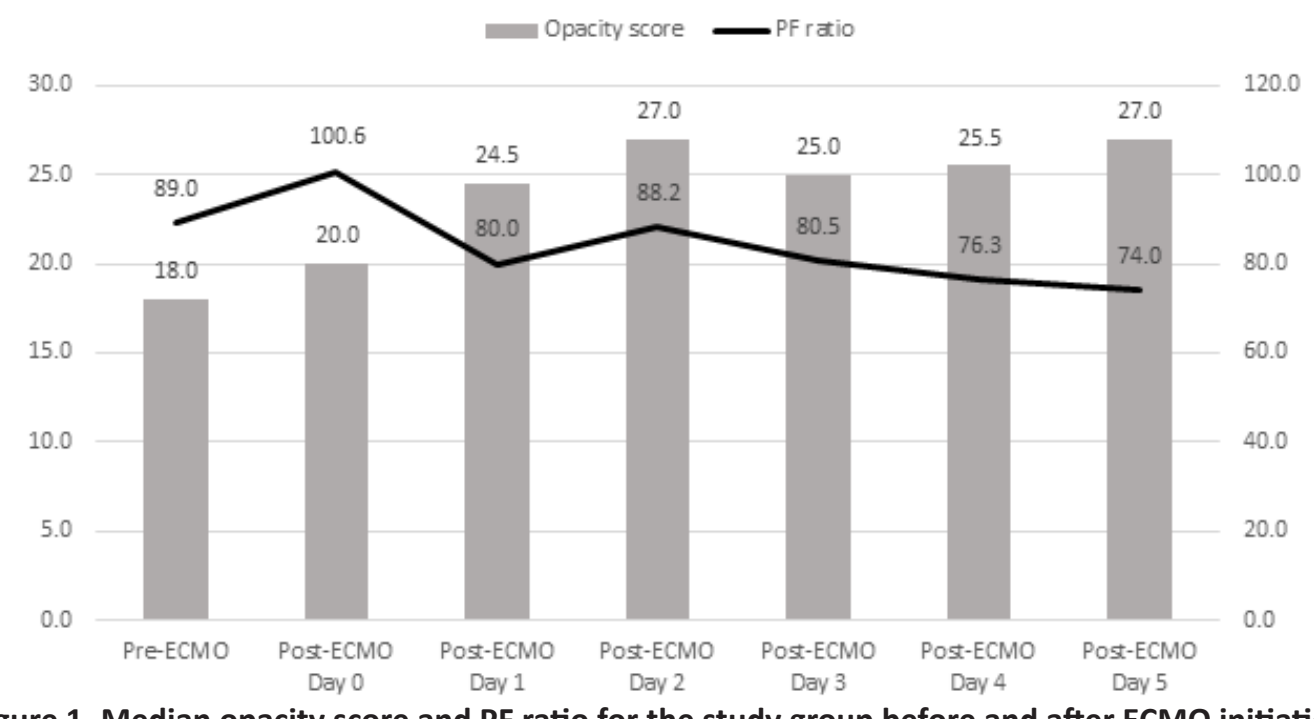

Figure 1. Median opacity score and PF ratio for the study group before and after ECMO initiation. 

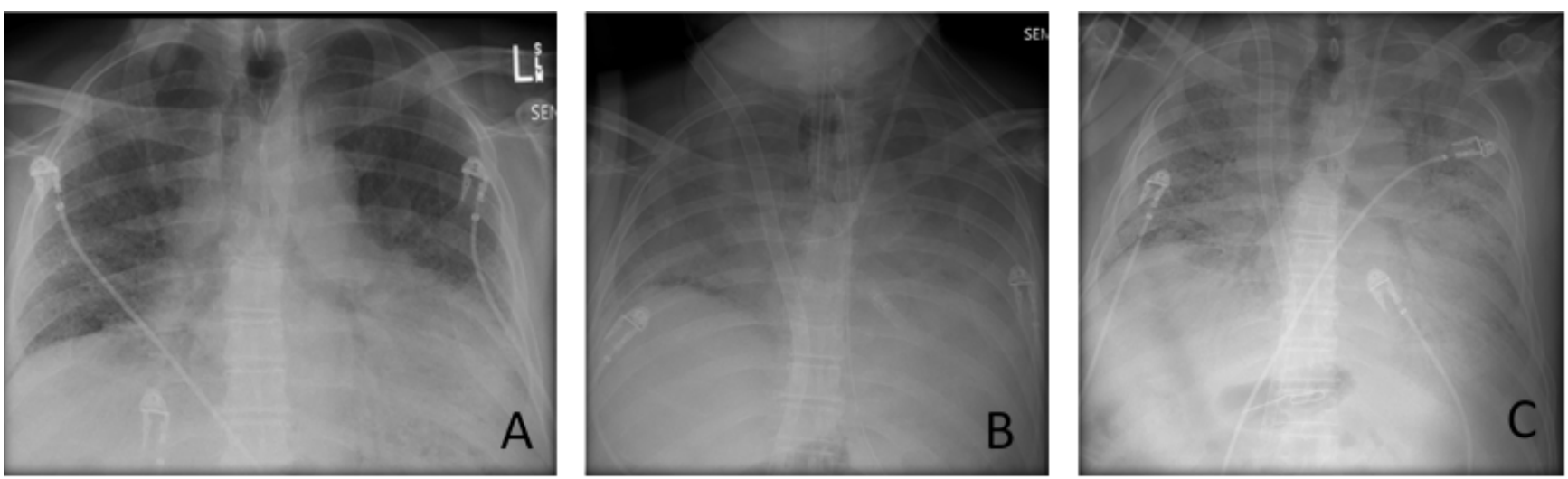

Fig. 2. 50/M patient with ARDS who was placed on ECMO for refractory hypoxemia. A: Chest radiograph before ECMO initiation. Right lung: Quality-2; Localization-3; Severity- 4; Lesion characteristics-0. Baseline score-9. Left lung: Quality-2; Localization-3; Severity-4; Lesion characteristics-0. Baseline score-9. B: Chest radiograph 48 hours after ECMO initiation. Right lung: Quality-2; Localization-3; Severity-4; Lesion characteristics- 2 (one point for each lower quadrant). Total score-11. Left lung: Quality-2; Localization-3; Severity-4; Lesion characteristics-3 (one point for each lower quadrant and one for the lateral upper quadrant). Total score-16. C: Chest radiograph 6 days after ECMO initiation. Right lung: Quality-2; Localization-3; Severity-4; Lesion characteristics-4 for each quadrant. Total score-25. Left lung: Quality-2; Localization-3; Severity-4; Lesion characteristics-4 for each quadrant. Total score - 25.

Overall, the two groups appeared to be similar concerning their pre-ECMO characteristics as well as postECMO course, although patients with a significant increase in opacity score had significantly lower median stay on ECMO. Additionally, the incidence of non-pulmonary organ dysfunction on ECMO was also similar for the two groups (Table 3).

The trends in the median opacity scores for the two groups revealed a statistically significant increase in the medianopacityscorestartingonpost-ECMODayl, which continued to remain significant after that (Figure 3).
Table 3. Development of new organ dysfunction among patients with and without the significant worsening in opacity score

\begin{tabular}{|c|c|c|c|}
\hline \multirow{2}{*}{ Variable } & \multicolumn{2}{|c|}{$\begin{array}{l}\text { Significant } \\
\text { worsening in } \\
\text { opacity score }\end{array}$} & \multirow{2}{*}{$\begin{array}{c}\text { Chi-square } \\
\text { P-value }\end{array}$} \\
\hline & $\begin{array}{c}\text { Yes } \\
(n=9)\end{array}$ & $\begin{array}{c}\text { No } \\
(n=24)\end{array}$ & \\
\hline Acute renal failure & $22.2 \%$ & $33.3 \%$ & 0.69 \\
\hline Shock & 55.6 & 70.8 & 0.41 \\
\hline Liver dysfunction & 11.1 & 29.2 & 0.28 \\
\hline Hematologic dysfunction & 77.8 & 79.2 & 0.93 \\
\hline
\end{tabular}

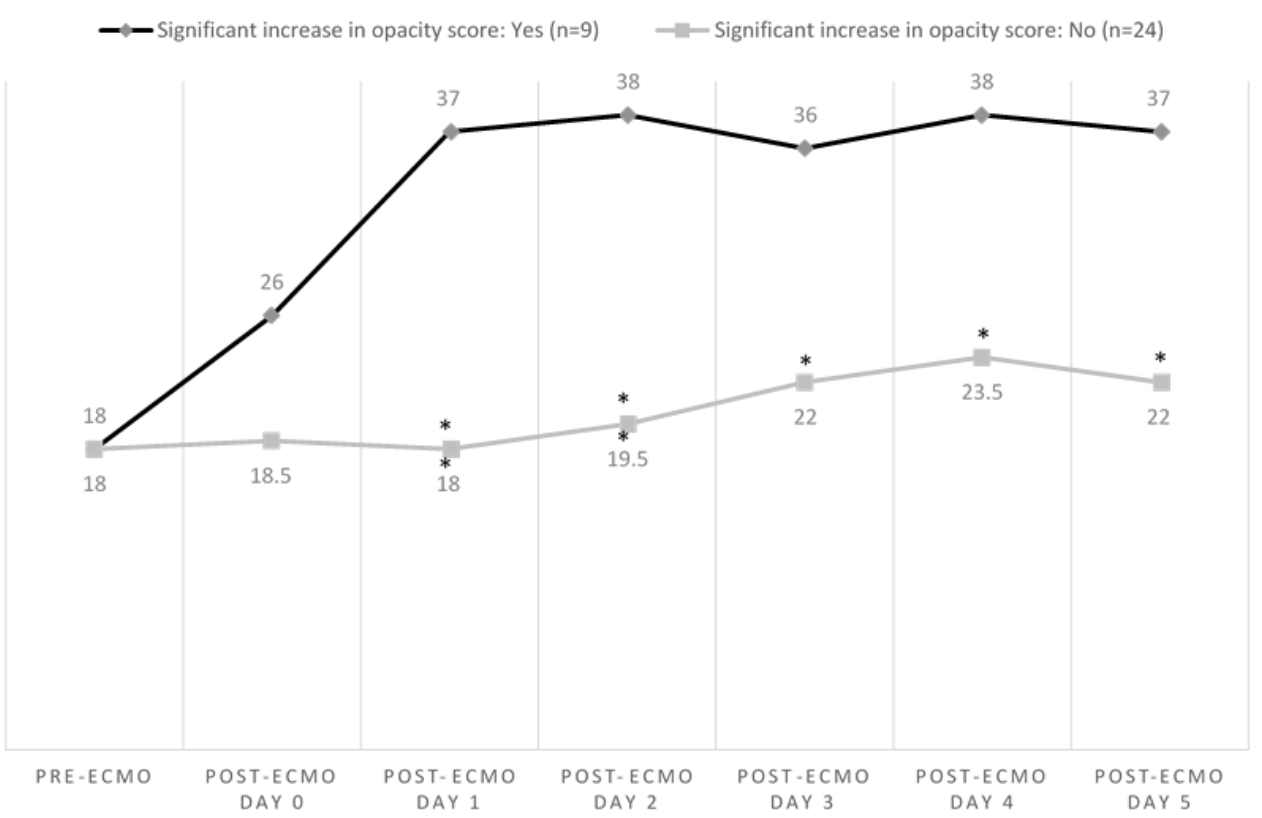

Fig. 3. Trends in the median opacity scores for the two groups. The difference between the two groups became statistically significant on Post-ECMO day 1 onwards. (Mann Whitney U test $*^{*} p<0.001 ; *_{p}<0.01$ ) 
The overall hospital survival was similar for the two groups.

\section{DISCUSSION}

The current study was conducted to assess the changes in functional pulmonary characteristics and radiologic changes after the initiation of ECMO support.

Routinely captured variables, namely, chest radiographs and the PF ratio were used to investigate these characteristics. The key benefit of using these two variables was their easy availability and consistent use in the routine daily care and clinical decision making among these patients. These characteristics should allow the results from the current analysis to be readily generalisable to other clinical settings.

A primary consideration during the study planning was the inherent challenge in evaluating the impact of $\mathrm{ECMO}$ on the functional and radiologic characteristics of lungs that are significantly abnormal in the first instance. Irrespective of whether the goals of ECMO was as a bridge to LT or bridge to recovery, patients needing ECMO support had profound background pulmonary impairment. To circumvent these issues, each patient was used as their own 'control' and utilised pre-ECMO characteristics as the baseline for comparisons.

While the PF ratio is widely used as a marker of functional assessment, no validated measure for objective assessment of chest opacities was available. It was necessary to quantify the extent and severity of new opacities that may have developed in the background of pre-existing lung disease necessitating ECMO support.

To this end, a dedicated scoring system to quantify the extent and severity of opacities on serial radiographs, was tested. Since this was a novel scoring system, two different thoracic radiologists independently scored the radiographs. Apart from the instrument having an excellent inter-observer agreement, a highly significant inverse association with the functional measure (PF ratio) supports the construct validity of the tool.

Based on the knowledge from earlier studies [3-10], mostly among paediatric populations, demonstrating an almost immediate increase in the pro-inflammatory cytokines with the initiation of ECMO, some degree of worsening of pulmonary status was anticipated.

A statistically significant increase in the opacity score for the entire study group confirmed that radiological worsening does indeed occur with ECMO initiation. In contrast, the functional assessment, as reflected by the oxygenation trends, was not statistically significant (Figure 1). It is noteworthy that the initiation of ECMO would be expected to improve the PF ratio given that a significant proportion of cardiac output is oxygenated by the circuit leading to higher $\mathrm{PaO}_{2}$, which in turn should permit a reduction in the $\mathrm{FiO}_{2}$. While this seemed to occur transiently in the immediate postECMO period (Figure 1), it was not sustained, and the $\mathrm{PF}$ ratio began to decline from post-ECMO Day 1 . In this context, a lack of improvement in the PF ratio may indicate a functional worsening after starting ECMO. Besides, other variables such as haemoglobin, cardiac output, size of the cannula, ECMO flows, recirculation with VV-ECMO, and fibrin or thrombus build-up in the oxygenator may impact systemic oxygenation and confound the association between PF ratio and pulmonary status.

While the current analysis substantiates the link between ECMO initiation and the worsening of opacities, it is unable to elucidate the putative mechanisms behind the association. The lack of difference in the need or quantity of transfusions before or after ECMO initiation between the two groups argues against transfusion-associated acute lung injury as the aetiology. Similarly, the median fluid balance was also similar among the two groups, which excludes volume overload as the culprit. While an inflammatory aetiology akin to acute lung injury as part of the ECMO associated SIRS [3-13] was considered, the lack of association with any of the other non-pulmonary organ dysfunction (Table 3) argues against this possibility. This is especially pertinent as the development of non-pulmonary organ dysfunction appeared to be correlated amongst each other. For example, patients with acute renal failure (ARF, 30.3\% of the study group), had significantly higher risk of hematologic dysfunction (100\% of patients with ARF vs $69.5 \%$ among those without ARF, $p=0.049$ ), hepatic dysfunction (60\% vs $8.7 \%, \mathrm{p}=0.002)$ and shock ( $90 \%$ vs $56.5 \%, \mathrm{p}=0.06)$. Regardless, the lack of association of worsening pulmonary opacities with other organ dysfunctions does not completely exclude an inflammatory aetiology as the timing and severity of the impact of cytokines on the pulmonary parenchyma may differ among organs. Physiologically, lungs are disadvantaged by way of being exposed to the full complement of the cardiac output as against the other organs, whereby their exposure to cytokines may be much higher. Additionally, lungs are the first organ to be exposed to 
the blood returning from the circuit, especially among patients on VV-ECMO, where these cytokines may initiate an inflammatory reaction. The almost ubiquitous ( $>93 \%$ of the study group) worsening of pulmonary opacities after ECMO initiation may indicate the same. In contrast, the SIRS response may be attenuated among other organs as they receive a fraction of the cardiac output, which has already passed through the pulmonary capillary bed. Furthermore, the immunoregulatory pathways in the pulmonary capillaries may attenuate the ongoing cytokine storm and reduce the risk of non-pulmonary organ dysfunction [14-17].

The comparative analysis among patients with and without a significant worsening of opacities did not reveal any difference in their presenting characteristics or outcomes. While the comparative analysis may have been underpowered given the small sample size, patients with significant worsening of opacities tended to have a higher proportion of interstitial lung disease (ILD) patients. It is indeed plausible that patients with background fibrotic lung diseases may be vulnerable to acute lung injury related to ECMO initiation. Patients with significantly worse opacities seemed to spend less time on the ECMO, although the eventual hospital survival was not different. The duration of ECMO support may be impacted by its goal as patients being bridged to LT often need to be supported for a shorter period as recovery usually takes longer. The goal for ECMO in the current study group was LT among the majority, which confounds the association of worsening pulmonary opacities with survival. Regardless of their pulmonary status, the high lung allocation score (usually the top one percentile of the waitlisted patients) among waitlisted ECMO patients ensures that they get prioritised for lung offers. It appears that ECMO associated pulmonary dysfunction may be more consequential among patients with a goal of recovery rather than those awaiting LT.

The current analysis has some limitations. The retrospective study design that does not allow causality assessment with an inability to account for unmeasured confounding variables. The pulmonary opacity score used to assess the serial radiologic changes has not been studied before, although we used two blinded readers to ensure the objectivity and reproducibility of the instrument. Also, the concurrent PF ratio assessments supported the construct validity of the instrument. While the sample size in the current analysis provided adequate information regarding serial chang- es in the daily assessments, comparative analysis of the two groups formed based on significant worsening of pulmonary opacities, seemed underpowered. More extensive studies are needed to elucidate predictors of worsening of pulmonary status and its impact among patients bridged to recovery. Finally, the current analysis is unable to evaluate the impact of management strategies for worsening oxygenation and pulmonary opacities and how they could have impacted the outcomes.

\section{CONCLUSIONS}

ECMO may be associated with a worsening of pulmonary status that starts early and tends to peak around 48 hours after initiation. The progression of opacities on radiographs seems to precede worsening in oxygenation that is typically seen beyond the 24 hours after ECMO initiation. Both the oxygenation and radiographic opacities tend to stabilise by the third postECMO day. Future research is needed to understand better the mechanistic pathways behind the worsening of pulmonary opacities, identify clinical, laboratory, and radiological variables that predict its development and evaluate its association with outcomes.

\section{CONFLICT OF INTEREST}

None to declare.

\section{REFERENCES}

1. Thiagarajan RR, Barbaro RP, Rycus PT, et al. Extracorporeal Life Support Organisation Registry International Report 2016. ASAIO J. 2017;63:60-7

2. Millar JE, Fanning JP, McDonald Cl, McAuley DF, Fraser JF. The inflammatory response to extracorporeal membrane oxygenation (ECMO): a review of the pathophysiology. Crit Care. 2016;20:387.

3. Wang S, Krawiec C, Patel S, et al. Laboratory evaluation of hemolysis and systemic inflammatory response in neonatal non-pulsatile and pulsatile extracorporeal life support systems. Artif Organs. 2015;39:774-81.

4. Rungatscher A, Tessari M, Stranieri C, et al. Oxygenator is the main responsible for leukocyte activation in an experimental model of extracorporeal circulation: a cautionary tale. Mediators Inflamm. 2015;2015:484979.

5. MclLwain RB, Timpa JG, Kurundkar AR, et al. Plasma concentrations of inflammatory cytokines rise rapidly during ECMO-related SIRS due to the release of preformed stores in the intestine. Lab Invest. 2010;90:128-39. 
Available online at: www.jccm.ro

6. Mildner RJ, Taub N, Vyas JR, et al. Cytokine imbalance in infants receiving extracorporeal membrane oxygenation for respiratory failure. Biol Neonate. 2005;88:321-7.

7. Graulich J, Sonntag J, Marcinkowski M, et al. Complement activation by in vivo neonatal and in vitro extracorporeal membrane oxygenation. Mediators Inflamm. 2002;11:69-73.

8. Doyle AJ, Hunt BJ. Current understanding of how extracorporeal membrane oxygenators activate haemostasis and other blood components. Front Med (Lausanne). 2018;5:352..

9. Sniderman J, Monagle P, Annich GM, MacLaren G. Hematologic concerns in extracorporeal membrane oxygenation. Res Pract Thromb Haemost. 2020;4:455-468.

10. Graulich J, Walzog B, Marcinkowski M, et al. Leukocyte and endothelial activation in a laboratory model of extracorporeal membrane oxygenation (ECMO) Pediatr Res. 2000;48:679-84.

11. Singer $M$, Deutschman CS, Seymour CW, et al. The Third International Consensus Definitions for Sepsis and Septic Shock (Sepsis-3). JAMA. 2016;315:801-10.
The Journal of Critical Care Medicine 2021;7(1) • 13

12. Beck L, Burg $M C$, Heindel W, Schülke C. Extracorporeal Membrane Oxygenation in Adults - Variants, Complications during Therapy, and the Role of Radiological Imaging. Rofo. 2017;189:119-27.

13. Sidebotham D, McGeorge A, McGuinness S, Edwards M, Willcox T, Beca J. Extracorporeal membrane oxygenation for treating severe cardiac and respiratory failure in adults: part 2-technical considerations. J Cardiothorac Vasc Anesth. 2010;24:164-72.

14. Moldoveanu B, Otmishi $P$, Jani $P$, et al. Inflammatory mechanisms in the lung. J Inflamm Res 2009;2:1-11.

15. Hayashi T, Beck L, Rossetto C, et al. Inhibition of experimental asthma by indoleamine 2,3-dioxygenase. J Clin Invest 2004;114:270-9

16. Raychaudhuri B, Fisher CJ, Farver CF, et al. Interleukin 10 (IL10)-mediated inhibition of inflammatory cytokine production by human alveolar macrophages. Cytokine. 2000;12:1348-55.

17. Pittet JF, Griffiths MJ, Geiser T, et al. TGF-beta is a critical mediator of acute lung injury. J Clin Invest 2001;107:1537-44. 